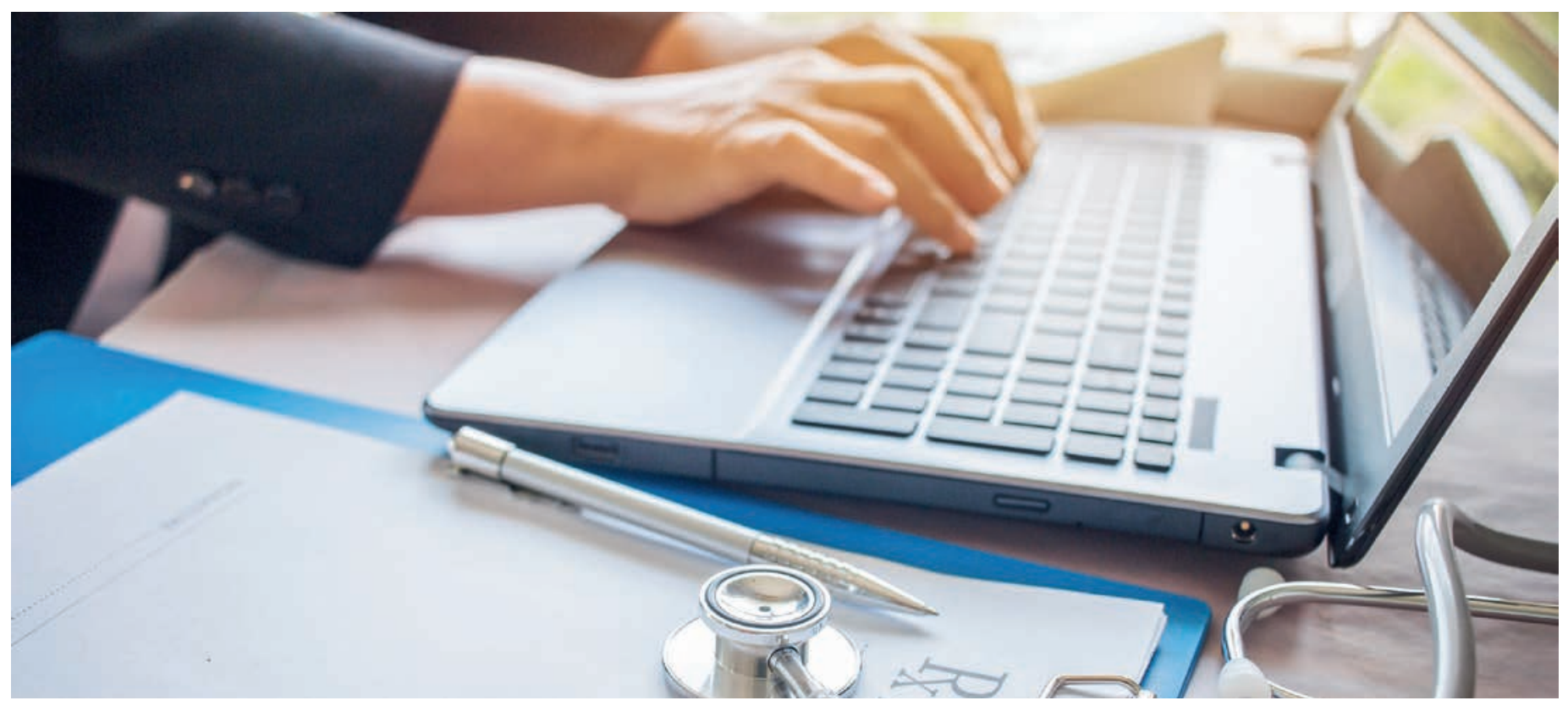

Digitalisierung soll Arbeitserleichterung im überlasteten Praxisalltag bieten. So ist im Bereich IV das bisherige medizinische Berichtsformular E 213 ab 1. Januar 2019 durch den neuen anwenderfreundlicheren "Ausführlichen ärztlichen Bericht» abgelöst worden. Nach einer Übergangsphase, während der beide Fassungen des internationalen Arztberichts verwendet werden können, wird ab dem 1. Juli 2019 nur noch das neue Berichtsformular zur Verfügung stehen.

Dr. med. Carlos Beat Quinto, Mitglied des FMH-Zentralvorstandes, Departementsverantwortlicher Public Health und Gesundheitsberufe

\title{
Der internationale Arztbericht wird digitalisiert
}

\section{Stefan Ritler}

Vizedirektor, Eidgenössisches Departement des Innern EDI, Bundesamt für Sozialversicherungen BSV

\section{Zweck des internationalen Arztberichts}

Der Informationsaustausch im Rahmen der Koordination der europäischen Sozialversicherungen sowie mit Vertragsstaaten, mit denen die Schweiz bilaterale Abkommen über die soziale Sicherheit abgeschlossen hat, erfordert einen internationalen Arztbericht. Das Formular E 213, welches hierfür bislang zur Verfügung stand, wird bis zum 1. Juli 2019 durch den neuen «Ausführlichen ärztlichen Bericht» ersetzt. Darin beantworten die behandelnden Ärztinnen und Ärzte standardisierte Fragen zur medizinischen Situation einer Person, die bei der Beantragung oder Überprüfung einer Invalidenrente zu klären sind. Damit ist auch der neue "Ausführliche ärztliche Bericht» eine notwendige Voraussetzung, um medizinische Informationen unter den Sozialversicherungen verschiedener Länder auszutauschen und internationale Rentenansprüche zu prüfen.

\section{Warum ein neues Formular?}

Derzeit entwickeln die Schweiz und die EU/EFTAMitgliedstaaten ein sicheres System für den elektronischen Austausch von Sozialversicherungsinformationen zwischen nationalen Institutionen, das sogenannte EESSI (Electronic Exchange of Social Security Information). Dabei ist es erforderlich, die alten 
europäischen Papierformen der E-Serie durch elektronische Formulare zu ersetzen. In diesem Zusammenhang erarbeitete ein Expertengremium aus der Schweiz und den EU-Mitgliedstaaten mit dem "Ausführlichen ärztlichen Bericht» ein moderneres, detaillierteres und anwenderfreundlicheres Formular für den internationalen Arztbericht. In einer abschliessenden Pilotphase testeten Mediziner aus Deutschland, Frankreich, Irland, Grossbritannien und Polen, die zuvor bereits mit dem Formular E 213 Erfahrungen gesammelt hatten, diesen unter realen Praxisbedingungen.

\section{Welche Versichertengruppe ist betroffen?}

Als neuer internationaler Arztbericht betrifft der «Ausführliche ärztliche Bericht» denselben Personenkreis wie zuvor das Formular E 213. Macht beispielsweise eine versicherte Person, welche vorgängig auch Versicherungszeiten in einem EU/EFTA- oder Vertragsstaat erworben hat, einen Leistungsanspruch gegenüber der IV geltend, wird der Anspruch nicht nur in der Schweiz, sondern parallel auch im entsprechenden Land geprüft.

\section{Welches sind die Vorteile des neuen elektronischen Formulars?}

Im Vergleich zur Vorgängerversion zeichnet sich der «Ausführliche ärztliche Bericht» durch eine erhöhte Anwenderfreundlichkeit aus. Vorteile ergeben sich insbesondere aus einem Paradigmenwechsel, weg von der klinischen Beurteilung hin zur Einschätzung der funktionellen Leistungsfähigkeit sowie zum expliziten Einbezug psychischer Erkrankungen. Dabei kommen eine moderne Terminologie und WHO-Standards (ICD-10) zum Einsatz.

Das Ausfüllen erfolgt elektronisch in ein vorstrukturiertes PDF-Formular. Zusätzlich zu den medizinischen Daten lassen sich Dokumente über Untersuchungsergebnisse als Anhang mitschicken. Das Formular ist "dynamisch" aufgebaut: Neben den allgemein notwendigen und zwingenden Pflichtfeldern müssen im Weiteren lediglich diejenigen Felder ausgefüllt werden, die auf Grundlage der zuvor gegebenen Antworten zur medizinischen Situation erscheinen.

Dadurch lässt sich der Zeitaufwand für das Ausfüllen des Formulars senken; ein Effizienzgewinn, der sich über die Möglichkeit der Zwischenspeicherung und automatischen Fehlerkorrektur zusätzlich steigern lässt. Neben dem reduzierten administrativen Aufwand wurden im Test v.a. auch die übersichtliche
Struktur des Formulars und die explizite Möglichkeit zur Beurteilung der Arbeitsfähigkeit positiv aufgenommen.

\section{Wo ist das neue Formular für den internationalen Arztbericht hinterlegt?}

Der internationale Arztbericht wird wie bisher angefordert. Die behandelnden Ärztinnen und Ärzte erhalten die Aufforderung zum Ausfüllen des Formulars und den nötigen Link auf die entsprechende Internetseite weiterhin von der IV-Stelle (IVST), die für einen angemeldeten Patienten, eine Patientin zuständig ist. Dabei ist das Formular sowohl über die Internet-Plattform für Ärzte (www.iv-pro-medico.ch) als auch die Website der AHV-IV-Informationsstelle (www.ahv-iv. ch/de) auffindbar.

\section{Werden die behandelnden Ärzte entschädigt?}

Die behandelnden Ärztinnen und Ärzte werden für das Ausfüllen des Berichtes genauso nach TARMED entschädigt wie für das Erstellen des bekannten im Inland verwendeten IV-Arztberichtes. Das Rechnungsformular ist auf der Internetseite der AHV-IV-Informationsstelle hinterlegt (www.ahv-iv.ch). Übersteigen die Kosten 500 Franken, muss die IVST vorgängig informiert werden.

\section{Übermittlung des Formulars und Datensicherheit}

Der behandelnde Arzt bzw. die behandelnde Ärztin füllt das Formular elektronisch im PDF-Format am PC aus (JavaScript muss zwingend aktiviert sein). Anschliessend wird das ausgefüllte Formular im PDF-Format ausgedruckt und zusammen mit den anderen Dokumenten, die zum Arztbericht gehören, per Briefpost an die zuständige IVST gesendet. Diese übermittelt es auf elektronischem Weg mit SWAP (Swiss Web Application Pension) an die Zentrale Ausgleichsstelle (ZAS) in Genf, welche es ihrerseits via geschlossenes EESSI-System an die involvierten ausländischen Stellen weiterleitet. Dank Verschlüsselung ist die Datensicherheit gewährleistet. Nur autorisierte Personen der zuständigen Einrichtungen der sozialen Sicherheit haben Zugang zu den medizinischen Daten.

Bildnachweis

(c) Samorn Tarapan | Dreamstime.com 